\title{
Angelicin inhibits the malignant behaviours of human cervical cancer potentially via inhibiting autophagy
}

\author{
YIRAN WANG ${ }^{1}$, YUEQI CHEN ${ }^{2}$, XUEDAN CHEN $^{3}$, YAN LIANG $^{4}$, DAPENG YANG ${ }^{1}$, \\ JIAO DONG ${ }^{1}$, NENG YANG ${ }^{1}$ and ZHIQING LIANG ${ }^{1}$
}

\begin{abstract}
Departments of ${ }^{1}$ Obstetrics and Gynecology, and ${ }^{2}$ Orthopedics, Southwest Hospital; ${ }^{3}$ Department of Medical Genetics, College of Basic Medical Science, Third Military Medical University, Chongqing 400038;

${ }^{4}$ Department of Respiratory Medicine, The General Hospital of PLA Rocket Force, Beijing 100088, P.R. China
\end{abstract}

Received September 14, 2018; Accepted July 24, 2019

DOI: $10.3892 /$ etm.2019.7985

\begin{abstract}
Angelicin is an active compound isolated from the Chinese herb Angelica archangelica, which has been reported to exert antitumor effects by inhibiting malignant behaviors in several types of tumor, including proliferation, colony formation, migration and invasion. However, the effects of angelicin on human cervical cancer cells is yet to be elucidated. The present study evaluated the antitumor effects of angelicin on cervical cancer cells. The results demonstrated that cervical cancer cells were more sensitive to angelicin than cervical epithelial cells. At its $\mathrm{IC}_{30}$, angelicin inhibited the proliferation of $\mathrm{HeLa}$ and $\mathrm{SiHa}$ cells by blocking the cell cycle at the G1/G0 phase and inhibiting other malignant behaviors, including colony formation, tumor formation in soft agar, migration and invasion. At the $\mathrm{IC}_{50}$, angelicin induced cell death potentially by promoting apoptosis. By identifying the hallmarks of autophagy, it was observed that angelicin treatment caused the accumulation of microtubule associated protein 1 light chain $3-\beta$ (LC3B) in the cytoplasm of HeLa and SiHa cells. Western blotting results demonstrated that cleaved LC3B-II and autophagy related proteins (Atg)3, Atg7 and Atg12-5 were upregulated following angelicin treatment. It was also determined that the phosphorylation of mTOR was induced by angelicin treatment. Furthermore, the inhibition of angelicin-induced mTOR phosphorylation did not disrupt its inhibitory effect on autophagy, indicating that angelicin inhibited autophagy in an mTOR-independent manner. Taken together, the present results suggested that angelicin regulated malignant behaviors in cervical cancer cells by inhibiting autophagy in an mTOR-independent manner. Findings suggested that autophagy might be a potential therapeutic target for cervical cancer.
\end{abstract}

Correspondence to: Professor Zhiqing Liang, Department of Obstetrics and Gynecology, Southwest Hospital, Third Military Medical University, 30 Gao-Tan-Yan Street, Shapingba, Chongqing 400038, P.R. China

E-mail: zhi_lzliang@163.com

Key words: angelicin, cervical cancer, mTOR, autophagy, malignant behaviors

\section{Introduction}

In less developed countries, cervical cancer is the second most commonly diagnosed cancer, with a high mortality rate (1). Early stage patients [International Federation of Gynaecology and Obstetrics (FIGO) stages I-IIA] may experience favourable outcomes by undergoing radical surgery or radiotherapy, as indicated by an overall 5 -year survival rate of $>65 \%(2,3)$. However, patients with later-stage disease, including stage IIB-IV, require more severe therapeutic strategies, including radiotherapy in addition to chemotherapy. The 5 -year survival rate for patients with stage IIB-III cancer is $25-30 \%$ (2,3). For stage IV cancer, the survival rate is $<15 \%(2,3)$ due to chemoresistance resulting in local recurrence or distant metastasis. Presently, chemotherapy is clinically employed as one of the most efficient strategies in the systematic treatment of cervical cancer. A combination of cisplatin with other chemotherapeutic drugs has remained the dominant systemic therapeutic modality for locally advanced and metastatic cervical cancer for several decades (4). However, chemoresistance limits the therapeutic effect of these chemoagent and frequently results in poor prognosis. Therefore, there is an urgent need for novel chemotherapeutic agents for use alone or in combination with a primary chemotherapeutic agent.

The natural molecule angelicin, 2-oxo-(2H)-furo(2,3-h)1-benzopyran, is one of the major active compounds isolated from the traditional Chinese herb Angelica archangelica. For decades, angelicin has been clinically used to exert therapeutic effects on various skin diseases, such as lichen planus, acting as a photosensitizer $(5,6)$. Angelicin exertsgenotoxic effects and thus induces cytotoxicity in several types of tumour and non-tumour cells (7,8). Mira and Shimizu (9) identified that angelicin causes cytotoxicity by inhibiting tubulin polymerization and histone deacetylase 8 activity in several types of tumour cells, including human hepatocellular carcinoma, rhabdomyosarcoma and colorectal carcinoma (9). To investigate the potential mechanism for proliferation inhibition, Wang et al (10) used liver cancer for therapeutic research both in vitro and in vivo. The study determined that the dose- and time-dependent apoptotic effect of angelicin is caused by the regulation of mitochondria, involving the P13K/AKT1 signalling pathway. Accordingly, 
angelicin affects physiological processes in both tumour and non-tumour cells.

Autophagy is a highly conserved multi-step lysosomal degradation process. Cellular components are sequestered in autophagosomes that subsequently fuse with lysosomes to degrade the contents (11). Accumulating evidence has established a close association between autophagy and tumour progression, with autophagy having different functions during tumour progression, including tumour suppression and enhancement $(5,6,12)$. Tsai et al (5) reported that the natural agent 1-(2-hydroxy-5-methylphenyl)-3-phenyl-1, 3 -propanedione, exerts growth-inhibiting effects by promoting the autophagy of HeLa cervical cancer cells. Li et al (6) determined that protein kinase $\mathrm{C}-\beta$ inhibited autophagy and consequently sensitized HeLa cells to chemotherapy. The study also reported that an increase in autophagy inhibited cell growth and induced apoptotic cell death (12). The dynamic role of autophagy in tumour progression has been the focus of research for potential therapeutics. However, further studies into strategies for controlling autophagy are required to increase understanding into the association between autophagy and tumour progression.

The growth-inhibiting and apoptosis-promoting effects of angelicin in several types of cancers have been previously reported (13). However, whether cervical cancer is chemosensitive to angelicin has not been demonstrated. Therefore, the present study used the human cervical carcinoma cell line, HeLa and the human cervical squamous cell carcinoma cell line, SiHa as in vitro models to determine the anticancer effects of angelicin. To evaluate its specific activity on cervical cancer cells, the non-tumour cervical epithelial cell line ECT1/E6E7 was also employed. The investigation primarily focused on the regulation of malignant behaviours by inducing or inhibiting autophagy in HeLa and SiHa. In addition, the effects of angelicin on autophagy and the potentially relevant mTOR signalling pathway were explored. The results of the present study may reveal the novel effects of angelicin as a chemotherapeutic strategy in certain types of cervical carcinomas.

\section{Materials and methods}

Cell culture and treatment. The human cervical carcinoma cell line, HeLa and the cervical squamous cell carcinoma cell line, SiHa was obtained from the American Type Culture Collection (accession no. HTB-35). The cervical epithelial cell line, ECT1/E6E7 was purchased from Jennio Biotech Co., Ltd. and used for identifying the difference of chemosensitivity between cancer cell lines and a non-tumor cell line. All cells were cultured at $37^{\circ} \mathrm{C}$ in a $5 \% \mathrm{CO}_{2}$ incubator in DMEM (Gibco; Thermo Fisher Scientific, Inc.) supplemented with $100 \mu \mathrm{g} / \mathrm{ml}$ streptomycin, $100 \mathrm{U} / \mathrm{ml}$ penicillin and $10 \% \mathrm{FBS}$ (Gibco; Thermo Fisher Scientific, Inc.). Cells were passaged every 3 days.

For identifying chemosensitivity, 0, 20, 40, 60, 80, 100, 120, 140, 160, 180 or $200 \mu \mathrm{M}$ angelicin (cat. no. A0956-10MG; Sigma-Aldrich; Merck KGaA) was added to the medium of HeLa or SiHa for 24 h. For 5-ethynyl-2'-deoxyuridine (Edu) staining, cell cycle distribution, colony formation, tumor formation in soft agar, migration and invasion assays, the IC30 of angelicin $(27.8 \mu \mathrm{M})$ was employed to evaluate the effects of angelicin on malignant behaviors. For carboxyfluorescein succinimidyl ester (CFSE)/propidium iodide (PI) or Annexin V FITC/PI double staining, the $\mathrm{IC}_{50}$ of angelicin was employed. For inhibiting the degradation of microtubule associated protein 1 light chain 3- $\beta$ (LC3B)-II, cells were pretreated with $10 \mu \mathrm{M}$ of chloroquine (cat. no. C6628; Sigma-Aldrich; Merck KGaA) for 6 h. For rapamycin (cat. no. V900930; Sigma-Aldrich; Merck KGaA) pretreatment, cells were pretreated with $1 \mu \mathrm{M}$ of rapamycin for $6 \mathrm{~h}$. Mock group containing vehicle only was considered as negative control in all the experiments.

Cell counting kit-8 (CCK-8) assay. To determine HeLa or SiHa cell viability, $5 \times 10^{3}$ cells were plated in 96 -well plates. The aforementioned treatment was administered and $10 \mu 1$ tetrazolium salt WST-8 (KeyGen Biotech. Co. Ltd.) was added to each well for a $4 \mathrm{~h}$ incubation at $37^{\circ} \mathrm{C}$. Optical density (OD) was measured at a wavelength of $450 \mathrm{~nm}$ using a microplate reader (Synergy 2 Multi-Mode Microplate Reader; BioTek Instruments, Inc.).

EdU staining. HeLa or $\mathrm{SiHa}$ cells were seeded at a density of $2 \times 10^{5}$ cells per well in 6-well plates supplemented with DMEM containing $50 \mu \mathrm{M}$ EdU (RiboBio Co. Ltd.). Following $2 \mathrm{~h}$ incubation at room temperature, cells were washed with ice-cold PBS and fixed with 4\% paraformaldehyde for $10 \mathrm{~min}$ at room temperature. EdU immunostaining was performed with Apollo staining reaction buffer followed by nuclei staining with Hoechst 33342 (cat. no. B2261; Sigma-Aldrich; Merck $\mathrm{KGaA}$ ) at final concentration of $10 \mu \mathrm{g} / \mathrm{ml}$ at room temperature for $10 \mathrm{~min}$. Stained cells were imaged under a X71 (U-RFL-T) fluorescence microscope (Olympus Corporation; magnification, $\mathrm{x} 40$ ).

PI staining. HeLa or SiHa cells were dissociated using $0.25 \%$ trypsin (Thermo Fisher Scientific, Inc.) and three time washed with PBS. Following the last wash, the cell pellet, which was centrifuged at $400 \times \mathrm{g}$ for $10 \mathrm{~min}$ at room temperature, was suspended and fixed in $70 \%$ ice-cold alcohol overnight at $4^{\circ} \mathrm{C}$. Cells were then washed in triplicate with ice-cold PBS and suspended in $400 \mu \mathrm{l}$ PI solution $(5 \mu \mathrm{g} / \mathrm{ml})$ for $30 \mathrm{~min}$ in the dark. Apoptotic cells were analyzed via flow cytometry using a 3 laser Navios flow cytometer (Beckman Coulter, Inc.) and analyzed using FlowJo software (FlowJo LLC; version 9).

Colony formation. HeLa or SiHa cells were seeded in 6-well plates at a density of 1,000 cells/well. Cells were then cultured at $37^{\circ} \mathrm{C}$ for 10 days until visible colonies appeared. Colonies were stained with $500 \mu \mathrm{l}$ Giemsa solution (Nanjing KeyGen Biotech Co., Ltd.) and incubated for $30 \mathrm{~min}$ at $37^{\circ} \mathrm{C}$. Colonies were then imaged using a X71 (U-RFL-T) fluorescence microscope (Olympus Corporation; magnification, $\mathrm{x} 40$ ).

Tumor formation in soft agar. To assess tumor formation in vitro, soft agar clonogenic assays were performed. Each well of a 6 -well plate was coated with $2 \mathrm{ml}$ of $0.5 \%(\mathrm{w} / \mathrm{v})$ low-melting agar (Sigma-Aldrich; Merck KGaA) in DMEM with $10 \%$ FBS. Cells were mixed and $5 \times 10^{3}$ cells in $2 \mathrm{ml}$ $0.3 \%$ low-melting agar with $10 \%$ FBS were added above the polymerized base solution. Plates were incubated $\left(37^{\circ} \mathrm{C}\right.$; 
$5 \% \mathrm{CO}_{2}$ ) for 14 days before colony number and diameter were quantified microscopically using a X71 (U-RFL-T) fluorescence microscope (Olympus Corporation; magnification, $\mathrm{x} 40$ ).

Scratch wound healing assay. HeLa or SiHa cells were seeded at a density of $1 \times 10^{6}$ in 6 -well plates and allowed to attach for $24 \mathrm{~h}$. When cell confluence reached $\sim 100 \%$, a scratch wound was subsequently introduced by scraping the cell monolayer with a $10 \mu \mathrm{l}$ sterile micropipette tip. Cells were then washed with PBS to remove unattached cells and incubated at $37^{\circ} \mathrm{C}$ for $24 \mathrm{~h}$ in medium containing $1 \%$ FBS. Cells were then imaged at the same site at 0 and $24 \mathrm{~h}$ following induction of the scratch using a X71 (U-RFL-T) fluorescence microscope (Olympus Corporation; magnification, $\mathrm{X} 40$ ).

Transwell invasion assay. HeLa or SiHa cells were dissociated with $0.25 \%$ trypsin and washed three times with ice-cold PBS. In the lower chamber, $500 \mu 1$ of DMEM supplemented with $10 \%$ FBS was added. A total of $200 \mu \mathrm{l}$ of cells at the concentration of $2 \times 10^{5} / \mathrm{ml}$ were seeded into the top chamber of transwell inserts containing $8 \mu \mathrm{M}$ pore polycarbonate filters (Corning Inc.) that had been precoated with Matrigel for $2 \mathrm{~h}$ at room temperature (BD Biosciences). The plate was incubated at $37^{\circ} \mathrm{C}$ for $24 \mathrm{~h}$. Experiments were performed in triplicate. Following $24 \mathrm{~h}$ of incubation, the cells on the upper membrane were removed and the invaded cells were stained with $0.25 \%$ crystal violet (Beyotime Institute of Biotechnology) at room temperature for $10 \mathrm{~min}$ then counted using a X71 (U-RFL-T) fluorescence microscope (Olympus Corporation; magnification, $\mathrm{x} 40)$.

CFSE/PI double staining. A total of $1 \times 10^{6} \mathrm{HeLa}$ or SiHa cells were seeded in 6-well plates and allowed to attach overnight. Then $100 \mu 1$ of CFSE fluorescent dye (Sigma-Aldrich; Merck KGaA) was added and incubated at $37^{\circ} \mathrm{C}$ for $15 \mathrm{~min}$. Supernatant was removed and cells were washed with DMEM without FBS. Following the aforementioned treatments, cells were incubated with PI to a final concentration of $5 \mu \mathrm{g} / \mathrm{ml}$ at room temperature for $10 \mathrm{~min}$. Cells were imaged using a X71 (U-RFL-T) fluorescence microscope (Olympus Corporation; magnification, $\mathrm{x} 40$ ).

Annexin V/PI double staining. HeLa or SiHa cells were dissociated using $0.25 \%$ Trypsin and washed three times with ice-cold PBS. Following the last wash, cells were suspended in PBS and the cell concentration was adjusted to $1 \times 10^{6} \mathrm{cell} / \mathrm{s} / \mathrm{ml}$. Cells were simultaneously stained with Annexin V-FITC (green fluorescence) and PI (red fluorescence), which allowed for the identification of intact cells (FITC $\left.-/ \mathrm{PI}^{-}\right)$, early apoptotic cells $\left(\mathrm{FITC}^{+} / \mathrm{PI}^{-}\right)$and late apoptotic cells $\left(\mathrm{FITC}^{+} / \mathrm{PI}^{+}\right)$. Samples were analyzed using the FACS LSRII flow cytometer (BD Biosciences) with FlowJo software (FlowJo LLC; version 9).

Immunofluorescence microscopy. HeLa or SiHa cells were plated in 6-well plates on coverslips and allowed to attach for $24 \mathrm{~h}$. Cells were fixed with $4 \%$ paraformaldehyde for $20 \mathrm{~min}$ and permeabilized with $0.2 \%$ Triton X-100 (Sigma-Aldrich; Merck KGaA) for $10 \mathrm{~min}$ at room temperature. Normal goat serum (5\%; Sigma-Aldrich; Merck KGaA) in PBS was used for unspecific blocking at room temperature for $30 \mathrm{~min}$. Cells were incubated with primary antibodies against LC3B (1:2,000; cat. no. ab48394; Abcam) at room temperature for $2 \mathrm{~h}$. Cells were then rinsed four times with PBS-Tween 20 and incubated with secondary antibodies produced in rabbit (1:500 in 0.5\% normal goat serum) conjugated with Alexa Fluor 488 for $1 \mathrm{~h}$ at room temperature. Cell nuclei were stained with DAPI (Sigma-Aldrich; Merck $\mathrm{KGaA}$ ) at room temperature for $10 \mathrm{~min}$. Images were captured with a X71 (U-RFL-T) fluorescence microscope (Olympus Corporation) at a magnification of x200.

Western blot analysis. Following the aforementioned treatments, HeLa or SiHa cells were lysed in chilled lysis buffer containing $50 \mathrm{mM}$ Tris- $\mathrm{HCl}$ (pH 7.4), $150 \mathrm{mM} \mathrm{NaCl}, 1 \%$ Triton X-100, $5 \mathrm{mM}$ EDTA, $1 \mathrm{mM} \mathrm{Na}_{3} \mathrm{VO}_{4}, 1 \mathrm{mM} \mathrm{NaF}$ and $10 \mu \mathrm{M}$ PMSF on ice for $10 \mathrm{~min}$. Supernatants were collected via centrifugation at $12,000 \mathrm{x}$ f for $10 \mathrm{~min}$ at $4^{\circ} \mathrm{C}$. The extracted protein concentration was measured using Bicinochoninic Acid kit (Sigma-Aldrich; Merck KGaA) for Protein Determination according to the manufacturer's protocol. For each sample, $20 \mu \mathrm{g}$ of total protein was loaded per lane and separated via SDS-PAGE on a $12.5 \%$ gel, then transferred onto a nitrocellulose membrane (EMD Millipore) followed by blocking using 5\% BSA (Sigma-Aldrich; Merck KGaA). The membrane was subsequently incubated with the following primary antibodies at a dilution of 1:1,000 overnight at $4^{\circ} \mathrm{C}$ : Rabbit anti-LC3B (cat. no. ab48394; Abcam), rabbit anti- $\beta$-actin (cat. no. ab8227; Abcam), rabbit anti-autophagy related protein (Atg)-3 (cat. no. ab108251; Abcam), rabbit anti-Atg7 (cat. no. ab133528; Abcam), rabbit anti- $\beta$-actin (cat. no. ab8227; Abcam), rabbit anti-Atg12-Atg5 (cat. no. orb375397; Biorbyt Ltd.), rabbit anti-mTOR (cat. no. ab2732; Abcam) and rabbit anti-mTOR (phospho S2448, cat. no. ab109268, Abcam). Membranes were subsequently incubated with horseradish peroxidase-conjugated secondary antibodies (goat anti-rabbit IgG H\&L antibody; cat. no. ab7090; 1:5,000; Abcam) for $2 \mathrm{~h}$ at room temperature. Enhanced chemiluminescence reagents (cat. no. PRN2232; GE Healthcare Bio-Sciences) were used to visualize protein bands.

Statistical analysis. Statistical analysis was performed with SPSS version 13.0 (SPSS, Inc.). Data were expressed as the mean \pm standard deviation. All experiments were repeated three times, independently. Comparisons between groups were assessed using a Student's t-test for two groups and one-way analysis of variance followed by Bonferroni post hoc analysis for multiple groups. $\mathrm{P}<0.05$ was considered to indicate statistical significance.

\section{Results}

Cervical cancer cells are more sensitive to angelicin than cervical epithelial cells. It has been reported that angelicin is cytotoxic to hepatic cancer cells (10). To investigate the effects of angelicin in cervical cancer cells (HeLa and SiHa), cell viability was determined using CCK-8 assay following exposure to a range of angelicin concentrations for $24 \mathrm{~h}$. For comparison, the sensitivity of cervical epithelial cells (ECT1/E6E7) to angelicin was also measured to evaluate the $30 \%$ inhibitory concentration $\left(\mathrm{IC}_{30}\right)$ and $50 \%$ inhibitory concentration $\left(\mathrm{IC}_{50}\right)$. The results 

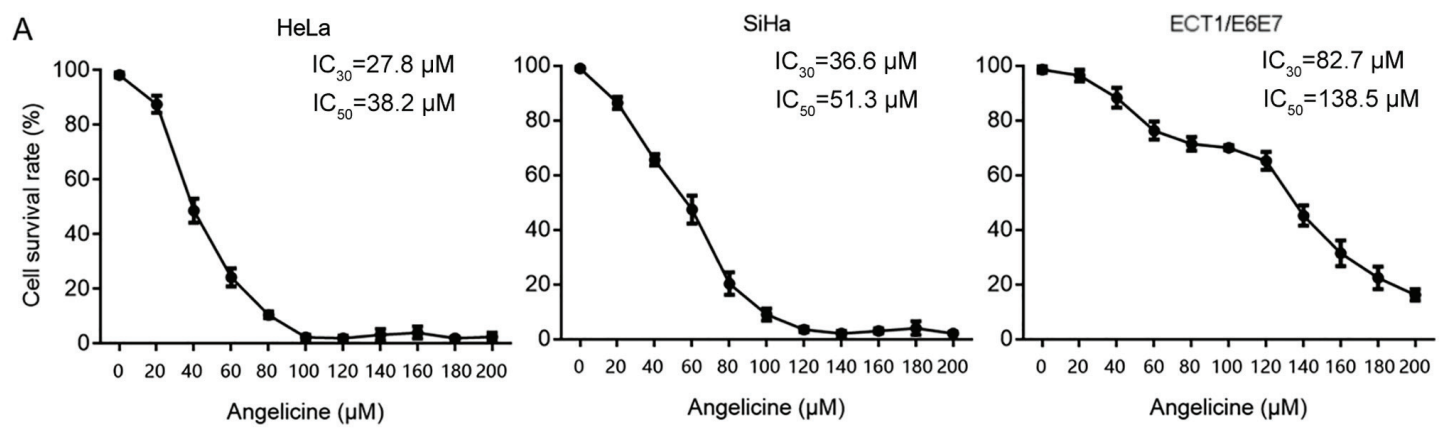

B

HeLa

$\mathrm{SiHa}$
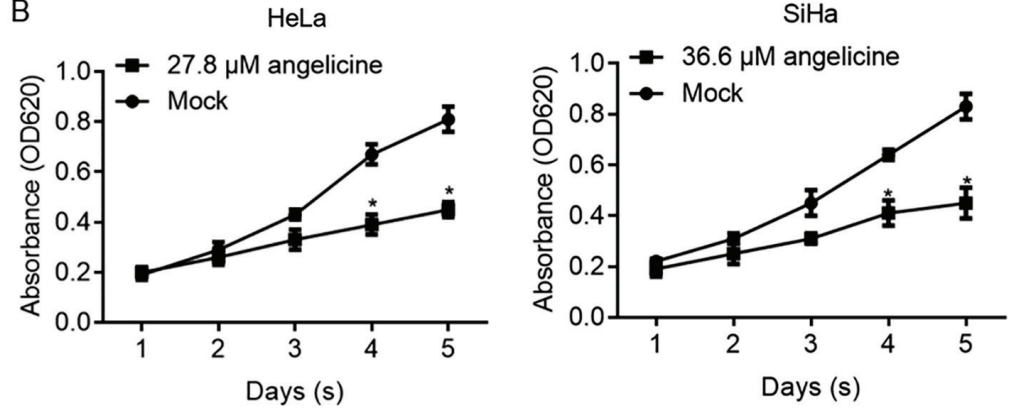

C

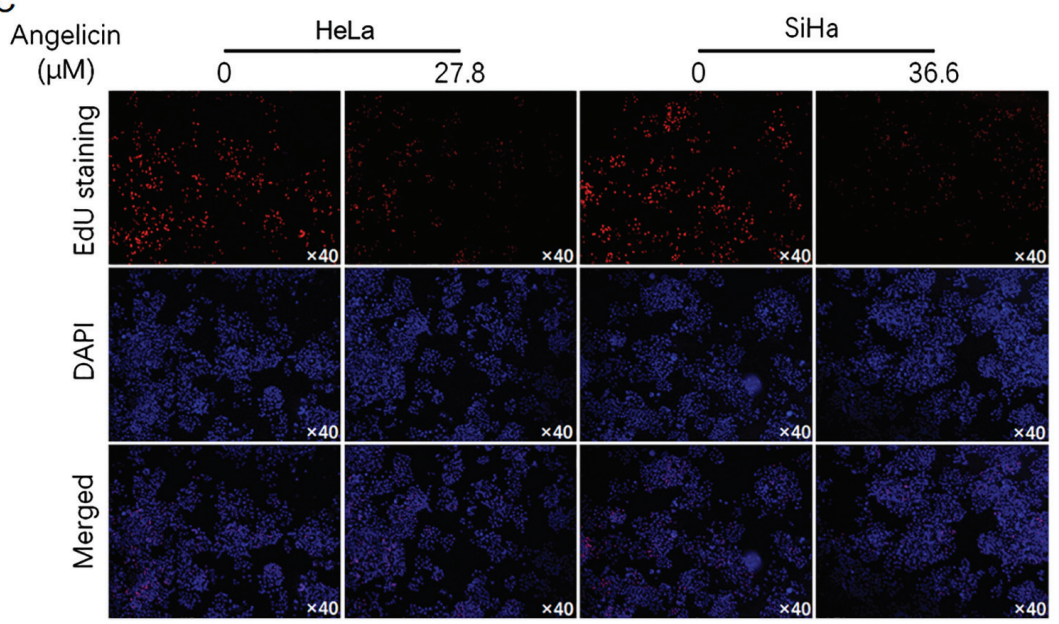

Figure 1. HeLa and SiHa cervical cancer cells were sensitive to angelicin treatment, whereas ECT1/E6E7 cervical epithelial cells were not. (A) Cell survival rate was measured by performing a cell counting kit-8 assay following angelicin treatment with indicated concentrations for $24 \mathrm{~h}$. The $30 \%$ inhibitory concentration $\left(\mathrm{IC}_{30}\right)$ and $50 \%$ inhibitory concentration $\left(\mathrm{IC}_{50}\right)$ were calculated accordingly. (B) Cell viability was measured following angelicin treatment at the $\mathrm{IC}_{30}$ from day 1 to 5. (C) EdU staining was performed to specifically label proliferating cells where blue staining represents cell nuclei and red staining represents proliferating cells. "P<0.05 vs. the mock group. Edu, 5-ethynyl-2'-deoxyuridine; OD, optical density.

revealed that $\mathrm{HeLa}\left(\mathrm{IC}_{30}, 27.8 \mu \mathrm{M} ; \mathrm{IC}_{50}, 38.2 \mu \mathrm{M}\right)$ and $\mathrm{SiHa}\left(\mathrm{IC}_{30}\right.$ $36.6 \mu \mathrm{M} ; \mathrm{IC}_{50}, 51.3 \mu \mathrm{M}$ ) cells were more sensitive to angelicin than ECT1/E6E7 cells ( $\mathrm{IC}_{30}, 82.7 \mu \mathrm{M} ; \mathrm{IC}_{50}, 138.5 \mu \mathrm{M}$; Fig. 1A). Cell viability was assessed on days $1-5$ following treatment with angelicin at the $\mathrm{IC}_{30}$. The results revealed that Angelicin treatment significantly inhibited HeLa and SiHa cell proliferation $(\mathrm{P}<0.05$ vs. mock group containing vehicle only; Fig. 1B). To confirm that the decrease in cell viability was due to a change in cell proliferation, EdU labelling of proliferating cells was performed. The results revealed that $\mathrm{HeLa}$ and $\mathrm{SiHa}$ cell treatment with angelicin at the $\mathrm{IC}_{30}$ substantially decreased the number of proliferating cells compared with the control (Fig. 1C).

Angelicin treatment inhibits the migration and invasion of cervical cancer cells. To determine whether angelicin inhibited cell proliferation by regulating cell cycle phase distribution, flow cytometry of PI-stained cells was performed to detect cell cycle entry following angelicin treatment. The ratio of cells in the G1 and G0 phases following angelicin treatment increased significantly compared with the mock group ( $\mathrm{P}<0.05$; Fig. 2A), whilst the ratio of cells in the $\mathrm{G} 2 / \mathrm{M}$ phases decreased substantially $(\mathrm{P}<0.05$; Fig. $2 \mathrm{~A})$. To investigate the effects of angelicin $\mathrm{IC}_{30}$ treatment on $\mathrm{HeLa}$ or $\mathrm{SiHa}$ cells, colony formation was assessed by seeding cells at low density to obtain single-cell-derived colonies as previously described (14). Mock-treated HeLa and SiHa cells formed single-cell-derived colonies (Fig. 2B). By contrast, angelicin treatment substantially decreased the colony formation ability of both HeLa and SiHa cells (Fig. 2B). The effect of angelicin on tumour formation in soft agar was investigated. Consistent with the effects exerted on colony formation $(>50 \mu \mathrm{M}$ in diameter), angelicin treatment markedly decreased the tumour 

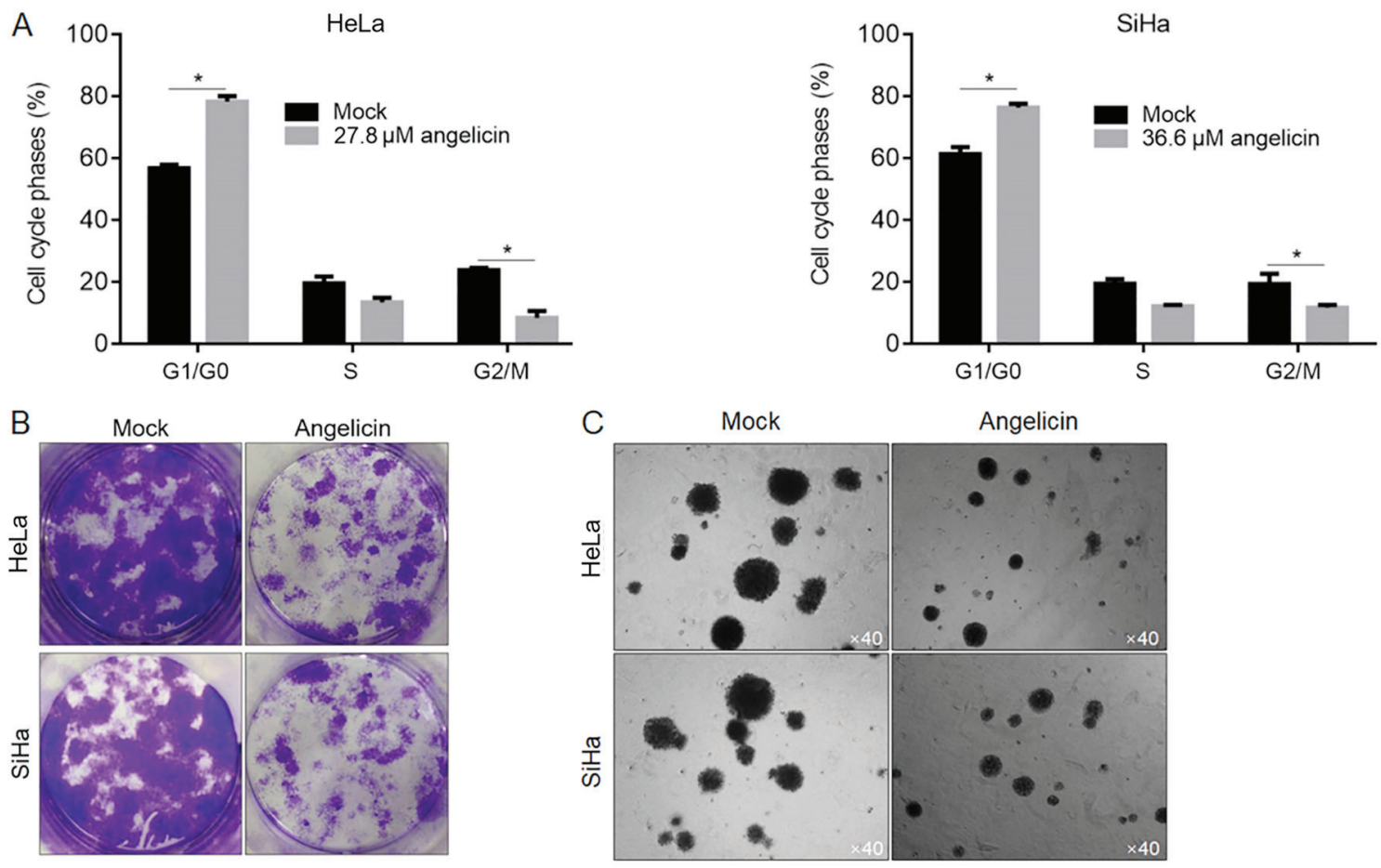

D
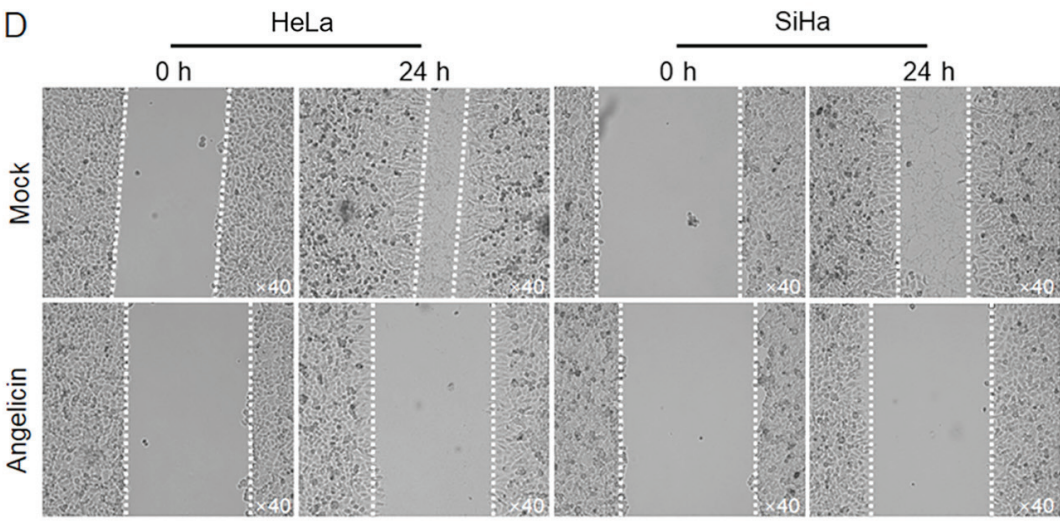

$\mathrm{E}$

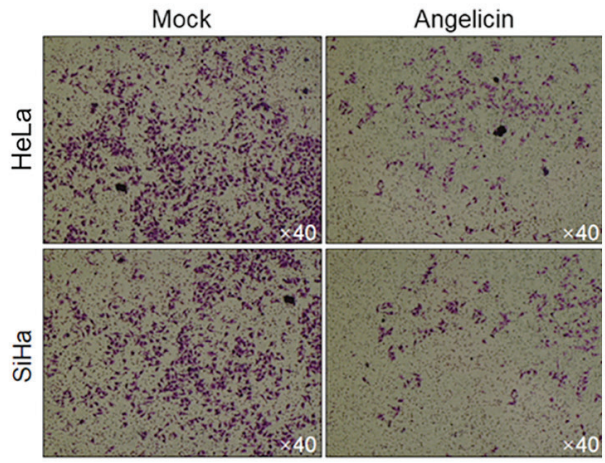

Figure 2. Angelicin inhibits the proliferation, colony formation, migration and invasion of cervical cancer cells. (A) Cell cycle distribution was measured by flow cytometry following propidium iodide staining. (B) Angelicin treatment reduced colony formation (C) tumour formation in soft agar (D), migration and $(\mathrm{E})$ invasion in $\mathrm{HeLa}$ and $\mathrm{SiHa}$ compared with the mock group. ${ }^{*} \mathrm{P}<0.05$ vs. mock group.

formation ability of the cells in soft agar compared with the control (Fig. 2C). Angelicin treatment substantially inhibited the migration and invasion of cells compared with mock treated cells (Fig. 2D and E).

Angelicin induces apoptotic cell death in HeLa and SiHa cells. The cytotoxic effect of angelicin was verified by a cytotoxicity assay with CFSE-labelled HeLa or SiHa cells. CFSE-positive cells demonstrated no significant change following angelicin treatment compared with mock treated cells (Fig. 3A). Following PI staining, it was observed that angelicin treatment markedly increased the number of PI-positive cells compared with mock treated cells, indicating that angelicin treatment increased the cell death rate (Fig. 3A). To further confirm that the increased cell death by angelicin treatment was due to induction of apoptosis, Annexin V/PI double-staining was performed. Angelicin treatment increased the apoptotic cell death rate (Annexin $\mathrm{V}^{+} / \mathrm{PI}^{-}$and Annexin $\mathrm{V}^{+} / \mathrm{PI}^{+}$) in both HeLa $(18.7 \pm 2.4 \%)$ and $\mathrm{SiHa}(16.9 \pm 3.1 \%)$ cells $(\mathrm{P}<0.05$; Fig. 3B $)$.
Taken together, the results indicated that angelicin treatment inhibited the malignant behaviours of HeLa and SiHa cervical cancer cells and induced apoptotic cell death.

Angelicin inhibits autophagy in cervical cancer cells. The chemotherapy-induced inhibition of cell viability, proliferation or cell death leading to the demise of cancer cells is mediated by autophagic pathways $(15,16)$. Therefore, the effects of angelicin treatment on autophagy were investigated in the present study. The regulatory effects of angelicin on autophagy were assessed by performing LC3B immunostaining. The results revealed that theLC3B-stained signal was greatly decreased following angelicin treatment for $24 \mathrm{~h}$ compared with the mock group in HeLa and $\mathrm{SiHa}$ cells (Fig. 4A). Chloroquine, a lysosome inhibitor inhibiting the fusion of autophagosomes and lysosomes and/or the activity of autolysosomes (17) was employed to accumulate LC3B-I and -II. Following the inhibition of LC3B degradation, the results revealed that angelicin treatment decreased the quantity of LC3B and cleaved 
A

HeLa

$\mathrm{SiHa}$

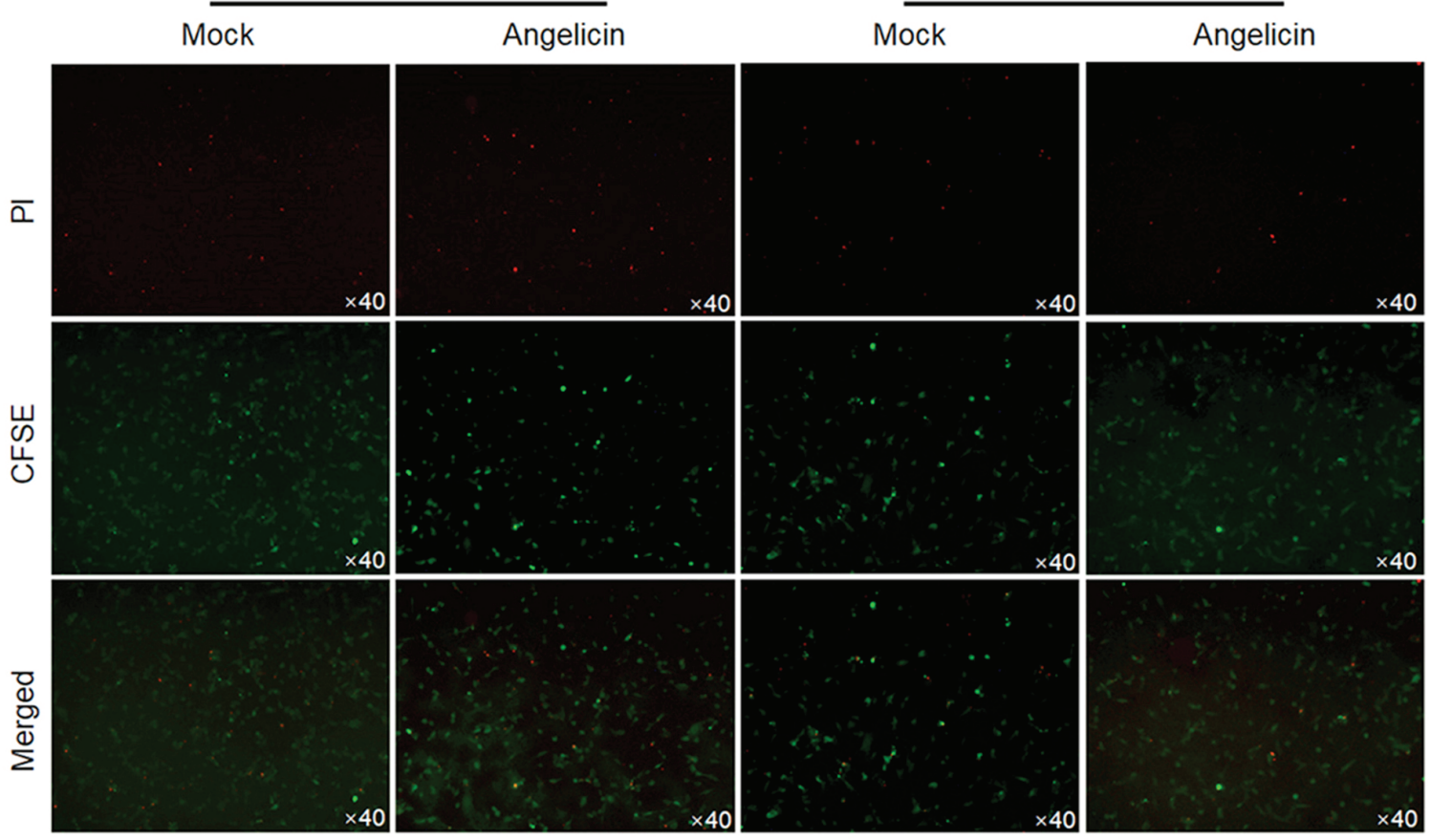

B

Mock

Angelicin
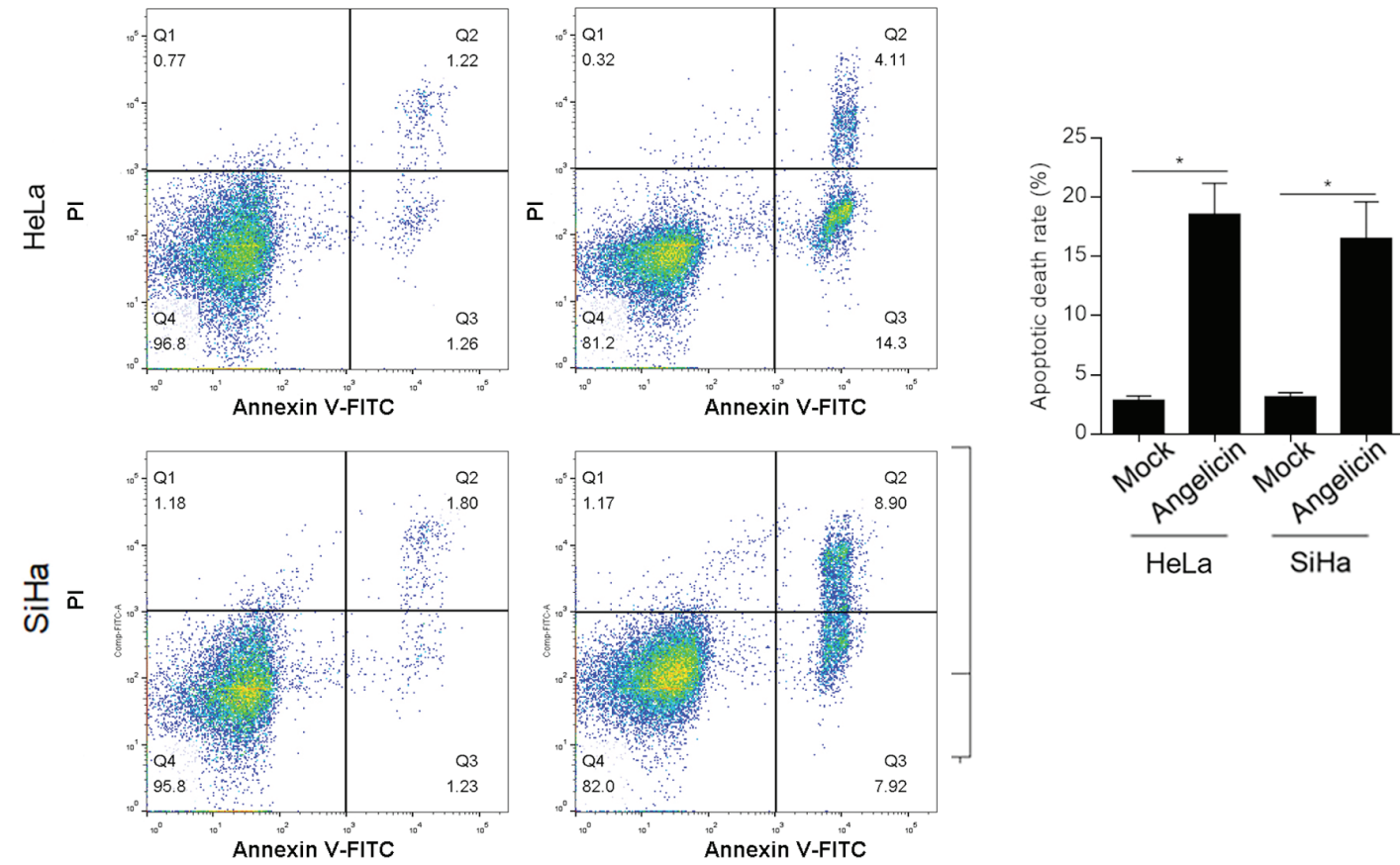

Figure 3. Angelicin treatment induced cervical cancer cell death via apoptosis. (A) Following staining with CFSE, HeLa or SiHa cells were treated with angelicin at the $\mathrm{IC}_{50}$ for $24 \mathrm{~h}$, followed by PI staining. The $\mathrm{CFSE}^{+} / \mathrm{PI}^{+}$subpopulation indicated dead cells. (B) Annexin V-FITC and PI double-staining was performed following $24 \mathrm{~h}$ treatment with angelicin at the $\mathrm{IC}_{50}$ with Annexin V-FITC $/ \mathrm{PI}^{-}$and Annexin V-FITC $/ \mathrm{PI}^{+}$cells. Representative histograms and quantification are presented. ${ }^{*} \mathrm{P}<0.05$ vs. mock group. CFSE, carboxyfluorescein succinimidyl ester; PI, propidium iodide.

LC3B-II compared with mock cells (Fig. 4B). The formation of an autophagosome involves the coordinated action of several Atg protein complexes (18-20). Thus, the expression of certain Atg proteins, including Atg3, Atg7 and Atg12-5, was determined via western blot analysis. Consistent with the change in LC3B, Atg3, Atg7 and Atg12-5 protein levels decreased following angelicin treatment compared with mock treatment (Fig. 4C).
Angelicin activates $m T O R$ phosphorylation and potentially regulates malignant behaviours by modulating autophagy in cervical cancer cells. mTOR is a central regulator of several physiological processes, including autophagy. Therefore, the current study assessed whether angelicin treatment regulated mTOR and thus affected autophagy. The results revealed that Angelicin treatment markedly increased the phosphorylation of mTOR in HeLa and SiHa cells, and decreased LC3B-II when 
A

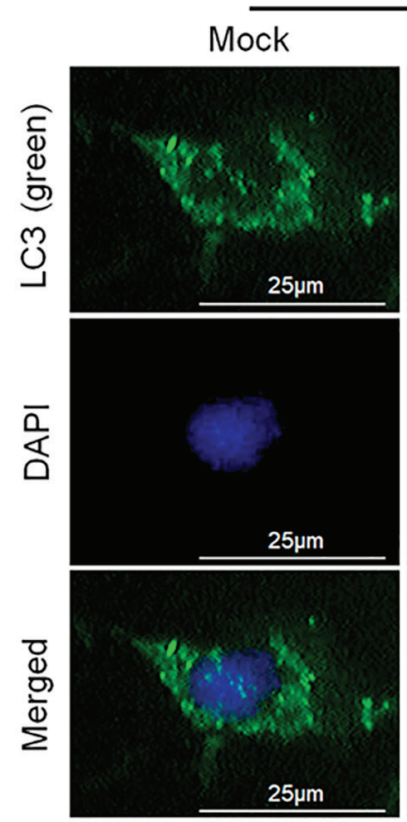

HeLa
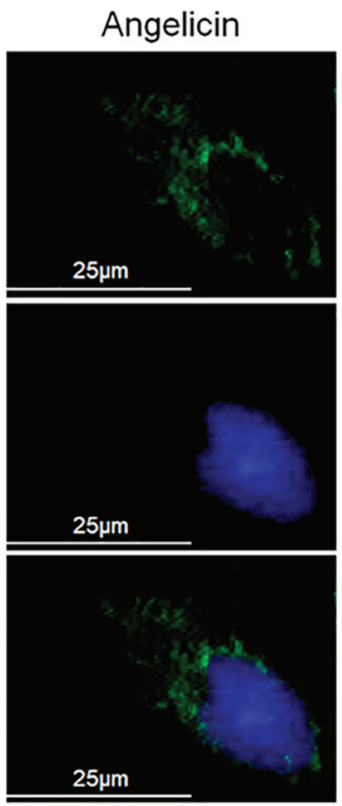

$\mathrm{SiHa}$
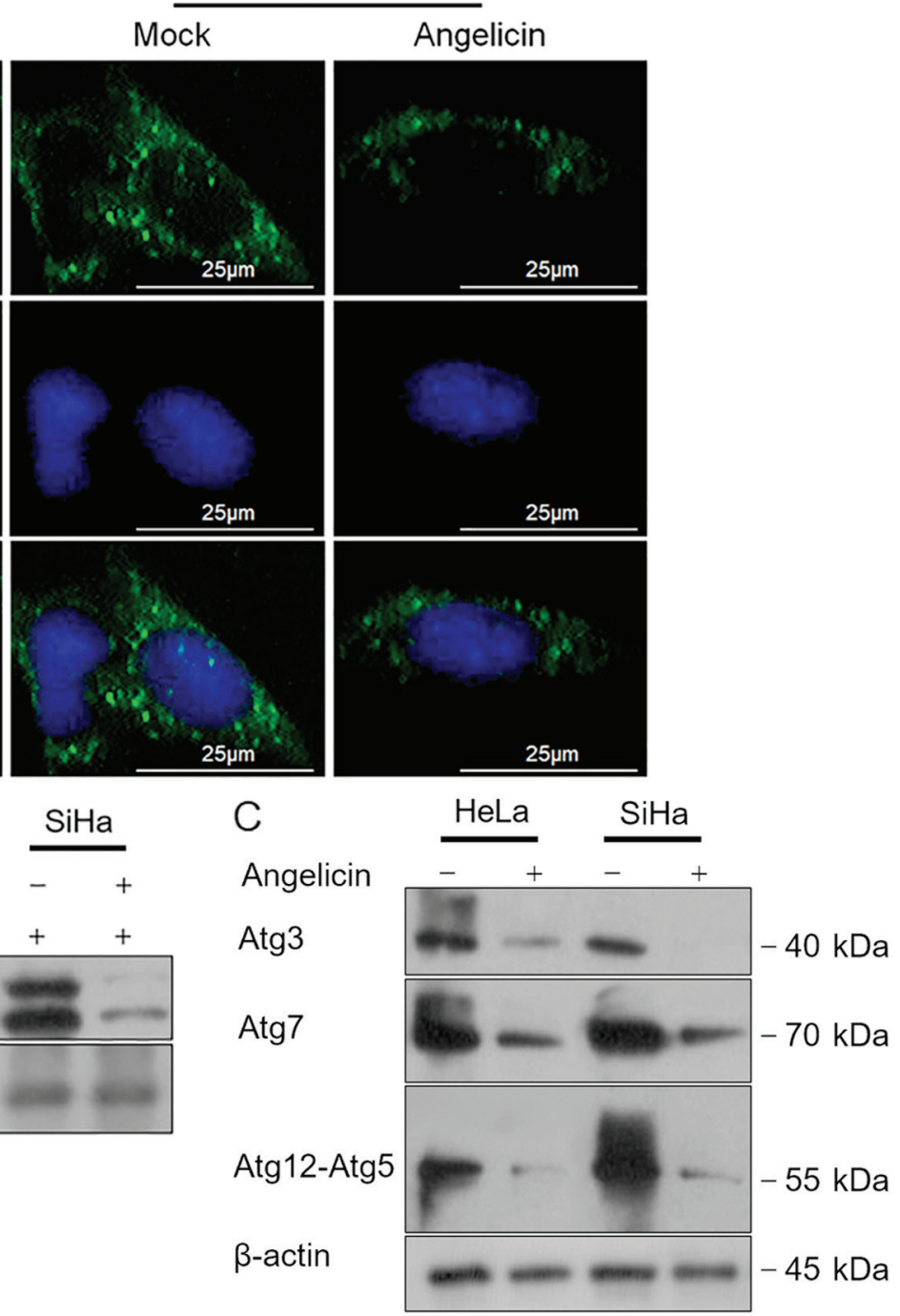

Angelicin
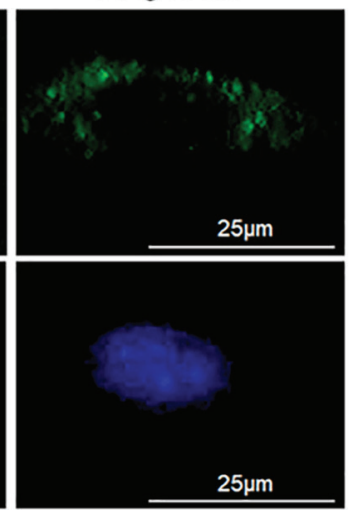

Angelicin

$\operatorname{Atg} 3$

Atg7

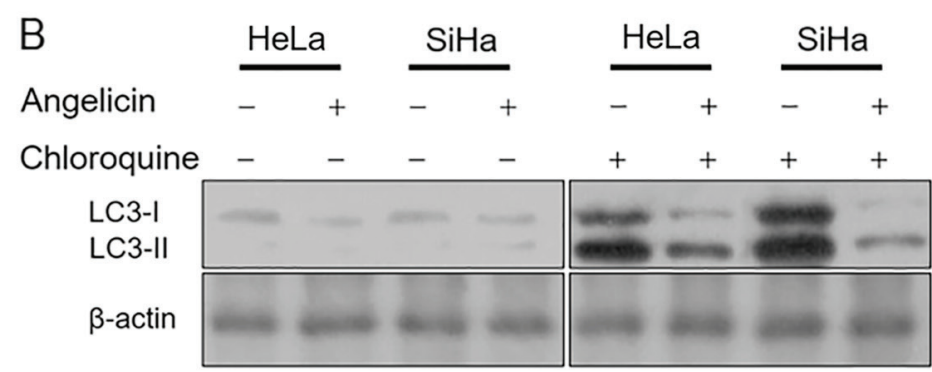

(A) Angelicin promoted autophagy in cervical cancer cells. (A) Immunofluorescence micrographs demonstrating LC3B staining (green) and nucleus staining (blue) following gangelicin treatment ( $27.8 \mu \mathrm{M}$ for HeLa, $36.6 \mu \mathrm{M}$ for SiHa). (B) Western blot analysis detected angelicin-induced LC3B expression in HeLa and SiHa cells. To inhibit the degradation of LC3B-II, cells were pre-treated with $5 \mu \mathrm{M}$ chloroquine for $6 \mathrm{~h}$. (C) Western blot analysis of Atg3, Atg7 and Atg12-Atg5 protein expression in both HeLa and SiHa cells. LC3B, microtubule associated protein 1 light chain 3- $\beta$; Atg, autophagy related proteins.

compared with mock treated cells (Fig. 5A). Following the addition of rapamycin, angelicin-induced mTOR phosphorylation was decreased, but the inhibitory effects of angelicin on autophagy were not fully reversed, indicating that mTOR might not be a direct target of angelicin, at least in part. To further confirm these results, cell viability and colony formation were measured following co-incubation with rapamycin and angelicin or rapamycin alone. As presented in Fig. 5B and C, angelicin treatment decreased the malignant behaviors of HeLa and SiHa cells, and indicated that rapamycin may exert its inhibitory effect in an mTOR-independent manner. Taken together, the results indicated that angelicin treatment regulated the phosphorylation of mTOR; however, this was not the main mechanism for affecting autophagy, which remains unknown.

\section{Discussion}

Angelicin has been previously reported to possess anticancer properties. In liver cancer, by activating the PI3K/AKT1 signalling pathway, angelicin treatment induced mitochondrial-dependent apoptotic cell death (10). Angelicin transcriptionally regulates members of the $\mathrm{Bcl}-2$ family of proteins that serve key roles in the regulation of the mitochondrial apoptotic pathway (21). The Bax/Bcl-2 ratio is therefore altered to cause mitochondrial destabilization, which leads to the release of proapoptotic factors (22). In the non-small cell lung cancer cell line and its sub-line (A549 and A549/D16, respectively) that exhibits multidrug resistance, angelicin treatment promoted chemotherapy-induced apoptotic cell death and sensitized A549/D16 cells to chemotherapy (23). However, the antitumor effects of angelicin in human cervical carcinoma, as well as the mechanisms underlying its actions, are largely unknown. D'Anqiolillo et al (24) reported that angelicin exerts cytotoxic activity on HeLa cells, but did not elucidate the exact mechanism by which this occurs (24). Therefore, the aim of the present study was to assess the effects of angelicin on the human cervical carcinoma cell lines, HeLa and SiHa, and to investigate the molecular mechanisms underlying its 
A

\begin{tabular}{lllll} 
A & \multicolumn{3}{c}{ HeLa } \\
\cline { 2 - 5 } RAPA & - & - & + & + \\
Angelicin & - & + & - & +
\end{tabular}

\begin{tabular}{llll}
\multicolumn{3}{c}{$\mathrm{SiHa}$} \\
\hline- & - & + \\
- & + & - & +
\end{tabular}
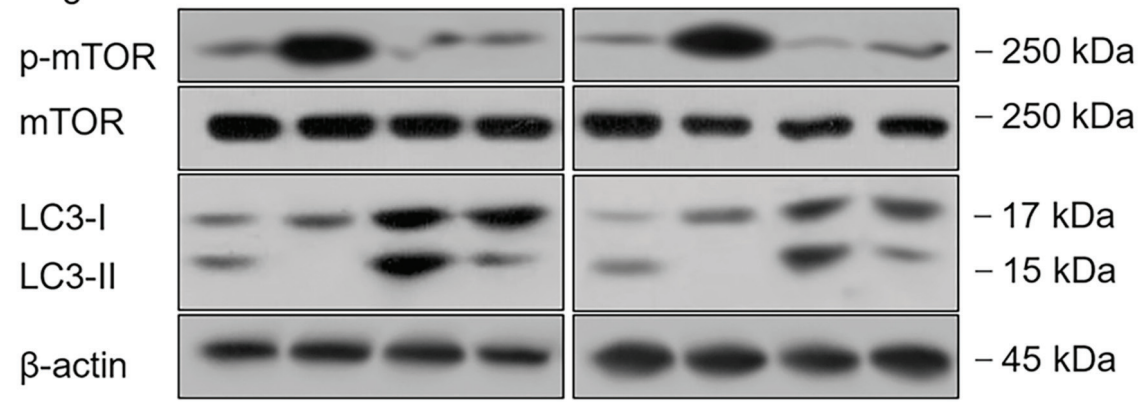

B

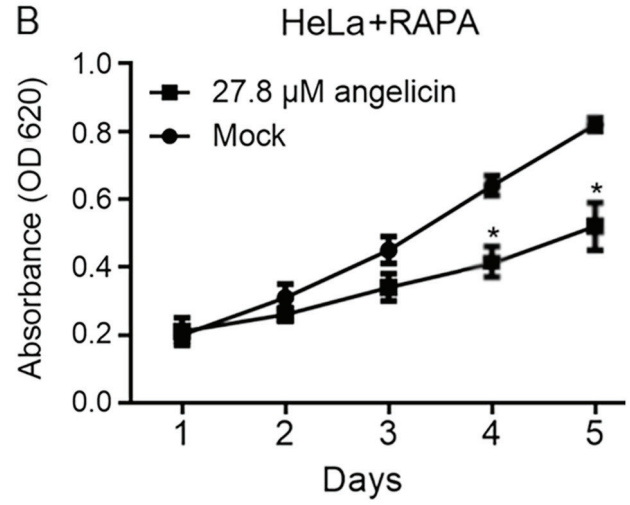

C
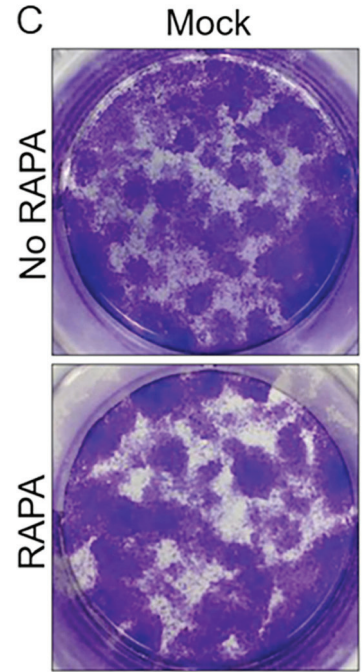

Angelicin

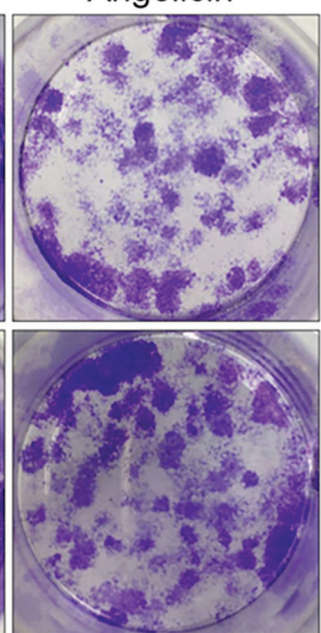

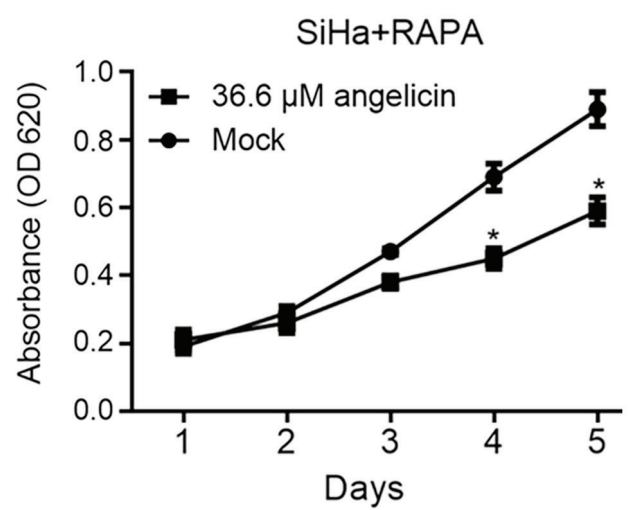

SiHa+RAPA

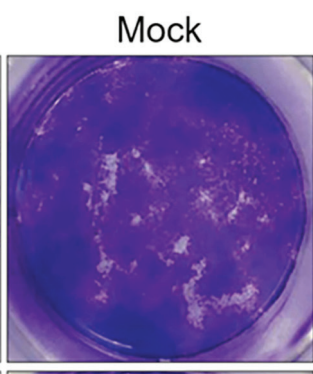

Angelicin

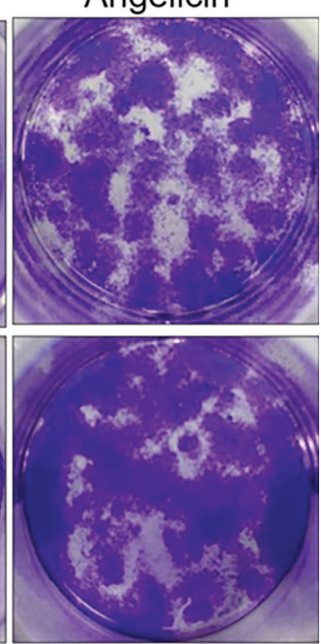

Figure 5. Angelicin activates mTOR phosphorylation and inhibits autophagy by an mTOR-independent pathway in cervical cancer cells. (A) Western blot analysis was performed following RAPA and angelicin co-treatment to identify whether autophagy stimulation by angelicin was dependent on mTOR phosphorylation. Following rapamycin and angelicin co-treatment, (B) cell viability and (C) colony formation were measured in HeLa and $\mathrm{SiHa}$ cells. * $\mathrm{P}<0.05$ vs. mock group. RAPA, rapamycin; p, phosphorylated; LC3B, microtubule associated protein 1 light chain 3- $\beta$; OD, optical density.

action. To determine whether cervical carcinomas were sensitive to angelicin, the non-tumour cervical epithelial cell line ECT1/E6E7 was also analyzed.

The present study determined that angelicin exerted antitumor effects on HeLa and SiHa cells but demonstrated no detectable cytotoxity to ECT1/E6E7 cells. Treatment of both HeLa and SiHa cells with angelicin at the $\mathrm{IC}_{30}$ suppressed malignant behaviours, including proliferation, colony formation, tumour formation in soft agar, migration and invasion. When evaluating migrating ability, medium containing $1 \%$ FBS was used instead of serum-free medium, which may be a limitation to the present study. Treatment with the $\mathrm{IC}_{50}$ of angelicin significantly induced cell death via apoptosis. Flow cytometry was employed to determine angelicin-induced apoptosis, however, a study limitation was that the detection of apoptosis markers, such as caspase-3, was not performed. The present study identified that angelicin treatment greatly inhibited autophagy by measuring hallmarks of autophagy, including LC3BI, LC3BII, Atg3, Atg7 and Atg12-5. Emerging evidence has indicated that interactions between autophagy and apoptosis occur via crucial proteins, including mTOR and Atgs (25). Through these regulatory mediators of crosstalk, 
cooperation between autophagy and apoptosis has been established. However, the present in vitro study requires in vivo research to further confirm the results gained.

mTOR is a critical regulator of autophagy that integrates nutrient signals and cytokines from different pathways, inhibiting autophagy and promoting cell growth (26). Signal starvation inhibits the phosphorylation of mTOR and initiates autophagy by forming theunc-51-like kinase complex, which comprises Atg13 and a protein tyrosine kinase 2-family interacting protein of $200 \mathrm{kDa}(27,28)$. The present study determined that angelicin treatment induced marked phosphorylation of mTOR without altering the total amount of mTOR. However, mTOR signalling has also been revealed to have no significant role in controlling autophagic flux (29), which may explain why rapamycin treatment failed to inhibit the effects of angelicin on autophagy regulation.

In conclusion, the present study demonstrated that angelicin treatment significantly inhibited malignant behaviours, including proliferation, colony formation, tumour formation, migration and invasion, in cervical cancer cells, potentially by inhibiting autophagy. Although angelicin treatment induced the phosphorylation of mTOR, its regulatory roles on autophagy and malignant behaviours were identified to be independent of mTOR signalling. Further studies are required to elucidate the exact molecular mechanisms underlying the regulatory role of angelicin on cervical cancer malignant behaviours. The results of the current study indicated that angelicin may have potential as a chemotherapeutic agent against cervical cancer.

\section{Acknowledgements}

The author would like to thank Mrs. Yun Bai (Third Military Medical University, Chongqing) for language editing.

\section{Funding}

No funding was received.

\section{Availability of data and materials}

The datasets generated and/or analyzed during the current study are available from the corresponding author on reasonable request.

\section{Authors' contributions}

YW, ZL and YC designed the experiments. XC, YL and DY performed cell culture and data analysis. YW wrote the manuscript. JD collected data and performed statistical analysis. NY is responsible for data collection. All authors read and approved the final manuscript.

\section{Ethics approval and consent to participate}

Not applicable.

\section{Patient consent for publication}

Not applicable.

\section{Competing interests}

The authors declare that they have no competing interests.

\section{References}

1. Torre LA, Bray F, Siegel RL, Ferlay J, Lortet-Tieulent J and Jemal A: Global cancer statistics, 2012. CA Cancer J Clin 65: 87-108, 2015.

2. Burger H, Loos WJ, Eechoute K, Verweij J, Mathijssen RH and Wiemer EA: Drug transporters of platinum-based anticancer agents and their clinical significance. Drug Resist Updat 14: 22-34, 2011.

3. Lilic V, Lilic G, Filipovic S, Milosevic J, Tasic M and Stojiljkovic M: Modern treatment of invasive carcinoma of the uterine cervix. J BUON 14: 587-592, 2009.

4. Rodríguez Villalba S, Díaz-CanejaPlanell C and Cervera Grau JM: Current opinion in cervix carcinoma. Clin Transl Oncol 13: 378-384, 2011.

5. Tsai JH, Hsu LS, Huang HC, Lin CL, Pan MH, Hong HM and Chen WJ: 1-(2-Hydroxy-5-methylphenyl)-3-phenyl1,3-propanedione induces g1 cell cycle arrest and autophagy in HeLa cervical cancer cells. Int J Mol Sci 17: E1274, 2016.

6. Li N and Zhang W: Protein kinase $\mathrm{C} \beta$ inhibits autophagy and sensitizes cervical cancer HeLa cells to cisplatin. Biosci Rep 37: BSR20160445, 2017.

7. Kavli G, Midelfart K, Raa J and Volden G: Phototoxicity from furocoumarins (psoralens) of Heracleum laciniatum in a patient with vitiligo. Action spectrum studies on bergapten, pimpinellin, angelicin and sphondin. Contact Dermatitis 9: 364-336, 1983.

8. Lampronti I, Bianchi N, Borgatti M, Fibach E, Prus E and Gambari R: Accumulation of gamma-globin mRNA in human erythroid cells treated with angelicin. Eur J Haematol 71: 189-195, 2003.

9. Mira A and Shimizu K: In vitro cytotoxic activities and molecular mechanisms of angelica shikokiana extract and its isolated compounds. Pharmacogn Mag 11 (Suppl 4): S564-S569, 2015.

10. Wang F, Li J, Li R, Pan G, Bai M and Huang Q: Angelicin inhibits liver cancer growth in vitro and in vivo. Mol Med Rep 16: 5441-5449, 2017.

11. Fang W, Shu S, Yongmei L, Endong Z, Lirong Y and Bei S: miR-224-3p inhibits autophagy in cervical cancer cells by targeting FIP200. Sci Rep 6: 33229, 2016.

12. Spanò V, Parrino B, Carbone A, Montalbano A, Salvador A, Brun P, Vedaldi D, Diana P, Cirrincione G and Barraja P: Pyrazolo[3,4-h]quinolines promising photosensitizing agents in the treatment of cancer. Eur J Med Chem 102: 334-351, 2015.

13. Bruni R, Barreca D, Protti M, Brighenti V, Righetti L, Anceschi L, Mercolini L, Benvenuti S, Gattuso G and Pellati F: Botanical sources, chemistry, analysis, and biological activity of furanocoumarins of pharmaceutical interest. Molecules 24: E2163, 2019.

14. Colter DC, Class R, DiGirolamo CM and Prockop DJ: Rapid expansion of recycling stem cells in cultures of plastic-adherent cells from human bone marrow. Proc Natl Acad Sci USA 97: 3213-3218, 2000.

15. Hu L, Sun S, Wang T, Li Y, Jiang K, Lin G, Ma Y, Barr MP, Song F, Zhang G and Meng S: Oncolytic newcastle disease virus triggers cell death of lung cancer spheroids and is enhanced by pharmacological inhibition of autophagy. Am J Cancer Res 5: 3612-3623, 2015.

16. Hanahan D and Weinberg RA: Hallmarks of cancer: The next generation. Cell 144: 646-674, 2011.

17. Trout JJ, Stauber WT and Schottelius BA: Increased autophagy in chloroquine-treated tonic and phasic muscles: An alternative view. Tissue Cell 13: 393-401, 1981.

18. Yang Z and Klionsky DJ: Mammalian autophagy: Core molecular machinery and signaling regulation. CurrOpin Cell Biol 22: 124-131, 2010.

19. Feng Y, He D, Yao Z and Klionsky DJ: The machinery of macroautophagy. Cell Res 24: 24-41, 2014

20. Tooze SA and Yoshimori T: The origin of the autophagosomal membrane. Nat Cell Biol 12: 831-835, 2010.

21. Adem J, Ropponen A, Eeva J, Eray M, Nuutinen U and Pelkonen J: Differential expression of Bcl-2 family proteins determines the sensitivity of human follicular lymphoma cells to dexamethasone-mediated and anti-BCR-mediated apoptosis. J Immunother 39: 8-14, 2016. 
22. Chen TC, Yu MC, Chien CC, Wu MS, Lee YC and Chen YC: Nilotinib reduced the viability of human ovarian cancer cells via mitochondria-dependent apoptosis, independent of JNK activation. Toxicol In Vitro 31: 1-11, 2016.

23. Hsieh MJ, Chen MK, Yu YY, Sheu GT and Chiou HL: Psoralen reverses docetaxel-induced multidrug resistance in A549/D16 human lung cancer cells lines. Phytomedicine 21: 970-977, 2014.

24. D'Anqiolillo F, Pistellia L, Noccioli C, Ruffoni B, Piaqqi S, Scarpato R and Pistelli L: In vitro cultures of Bituminariabituminosa: Pterocarpan, furanocoumarin and isoflavone production and cytotoxic activity evaluation. Nat Prod Commun 9: 477-480, 2014.

25. Li M, Gao P and Zhang J: Crosstalk between autophagy and apoptosis: Potential and emerging therapeutic targets for cardiac diseases. Int J Mol Sci 17: 332, 2016.

26. Kamada Y, Yoshino K, Kondo C, Kawamata T, Oshiro N, Yonezawa K and Ohsumi Y: Tor directly controls the Atg1 kinase complex to regulate autophagy. Mol Cell Biol 30: 1049-1058, 2010 .
27. Hara T and Mizushima N: Role of ULK-FIP200 complex in mammalian autophagy: FIP200, a counterpart of yeast Atg17? Autophagy 5: 85-87, 2009.

28. Mizushima N: The role of the Atg1/ULK1 complex in autophagy regulation. CurrOpin Cell Biol 22: 132-139, 2010.

29. Choi H, Merceron C, Mangiavini L, Seifert EL, Schipani E, Shapiro IM and Risbud MV: Hypoxia promotes noncanonical autophagy in nucleus pulposus cells independent of MTOR and HIF1A signaling. Autophagy 12: 1631-1646, 2016.

(i) This work is licensed under a Creative Commons Attribution-NonCommercial-NoDerivatives 4.0 International (CC BY-NC-ND 4.0) License. 